\title{
468.
}

\section{ADDITION TO SECOND NOTE ON THE LUNAR THEORY.}

[From the Monthly Notices of the Royal Astronomical Society, vol. xxvII. (1866-1867), pp. 267-269.]

Writing as in my Second Note, Monthly Notices, Vol. xxv., pp. 203-207 (May 1865), [466], for the Moon,

$$
\begin{aligned}
& a, \text { the mean distance, } \\
& e \text {, the excentricity, } \\
& \gamma \text {, the tangent of the inclination, } \\
& l \text {, the mean longitude, } \\
& c, \text { the mean anomaly, } \\
& g \text {, the mean distance from node, }
\end{aligned}
$$

I obtained by the ordinary method of the variation of the elements, from the constant term of $R$ and the term involving $\cos (2 c-2 g)$, the following expressions of the variations,

$$
\begin{array}{lccc}
\delta a=0, & & \\
\delta e=-\frac{5}{8} \gamma^{2} e & \cos & 2 c-2 g \\
\delta \gamma=+\frac{5}{8} \gamma e^{2} & \# & 2 c-2 g \\
\delta c=+\frac{5}{8} \gamma^{2} & \text { sin } & 2 c-2 g \\
\delta g=+\frac{5}{8} e^{2} & \# & 2 c-2 g \\
\delta l=+\frac{5}{16} \gamma^{2} e^{2} & \# & 2 c-2 g,
\end{array}
$$

viz. if in the elliptic expressions of the radius vector, longitude, and latitude, we apply to $a, e, \gamma, c, g, l$, the foregoing increments, we obtain to the fourth order in $(e, \gamma)$ the portions independent of $m$ in the expressions of the radius vector, latitude, 
and longitude. I wish to notice that the results, to the very limited extent to which they go, agree with those obtained by M. Delaunay in his "Théorie du Mouvement de la Lune," from his 49th operation, the object of which is to take away the term (63) of $R$, that is the term involving $\cos (2 c-2 g)$. The formulæ (see vol. I. p. 788), taken only to the necessary degree of approximation are

$$
\begin{array}{rcccc}
a & \text { replaced by } & a, \\
e^{2} & \# & e^{2}-5 \gamma^{2} e^{2} & \cos & 2 g, \\
\gamma^{2} & \# & \gamma^{2}+\frac{5}{4} \gamma^{2} e^{2} & \# & 2 g, \\
l & \# & l-\frac{5}{2} \gamma^{2} & \sin & 2 g, \\
h+g+l & \# & h+g+l+\frac{5}{4} \gamma^{2} e^{2} & \# & 2 g, \\
h & \# & h+\frac{5}{8} e^{2} & \# & 2 g,
\end{array}
$$

which, observing that

$$
\begin{aligned}
& \gamma(\text { Del. })=\frac{1}{2} \gamma \text { (for present purpose), } \\
& l \quad=c \\
& g+l \quad=g \\
& h+g+l=l
\end{aligned}
$$

and therefore

$$
g \quad=-(c-g) \text {, }
$$

become

$$
\begin{array}{rrrrr}
a & \text { replaced by } a, \\
e^{2} & \# & e^{2}-\frac{5}{4} \gamma^{2} e^{2} & \cos & 2 c-2 g, \\
\gamma^{2} & \# & \gamma^{2}+\frac{5}{4} \gamma^{2} e^{2} & \# & 2 c-2 g, \\
c & \# & c+\frac{5}{8} \gamma^{2} & \sin & 2 c-2 g, \\
l & \# & l-\frac{5}{16} \gamma^{2} e^{2} & , & 2 c-2 g, \\
l-g & \# & l-g-\frac{5}{8} e^{2} & \# & 2 c-2 g,
\end{array}
$$

the last of which may be changed into

$$
g \quad \Rightarrow \quad g+\frac{5}{8} e^{2} \quad, 2 c-2 g,
$$

or if the new values of $a, e, \gamma, c, g, l$, are called $a+\delta a, e+\delta e, \gamma+\delta \gamma, c+\delta c, g+\delta g, l+\delta l$, then the increments $\delta a, \delta e, \delta \gamma, \delta c, \delta g, \delta l$, have the values given above. The process of my Second Note, taken as a first transformation, has in fact the object of removing the term $\cos (2 c-2 g)$, and to the degree of approximation regarded, the result is not affected by the previous transformations, or by the substitution, t. II. p. 800 , introducing for $a, e, \gamma$, their standard elliptic values. 